Medicina Baseada Euidencias

\section{HÁ BENEFÍCIO PARA PACIENTES COM INSUFICIÊNCIA CORONARIANA E REGURGITAÇÃO MITRAL ISQUÊMICA AO SEREM SUBMETIDOS À CORREÇÃO DE VALVA MITRAL ASSOCIADA À REVASCULARIZAÇÃO MIOCÁRDICA?}

P: Coronariopatia e regurgitação mitral isquêmica.

I: Revascularização do miocárdio e cirurgia corretiva de valva mitral.

C: Revascularização do miocárdio.

O: Mortalidade e reintervenção a partir de 1,5 anos.

\section{Introdução}

Coronariopatia e regurgitação mitral funcional isquêmica estão fortemente associadas a pobres resultados em pacientes com doença arterial coronariana avançada. Opções de tratamento cirúrgico incluem revascularização isoladamente ou concomitante à valvuloplastia ou substituição da valva mitral'. O impacto da associação do reparo da valva mitral à revascularização em pacientes com regurgitação mitral isquêmica continua não elucidado².

\section{Estratégia de Busca}

Com o intuito de analisar o que há na literatura sobre o real benefício da intervenção em valva mitral além da revascularização, foi utilizada a seguinte estratégia de busca, na base Medline: [Coronary artery bypass] AND [Mitral valve insufficiency / Surgery OR Mitral valve / Surgery OR Heart valve prosthesis implantation] Limits: Clinical Trials OR Comparative study.

\section{Resultado da busca}

Foram recuperados 285 trabalhos. Por meio da análise do título e resumo foram selecionados, de acordo com o PICO, nove artigos que respondiam à questão e que, portanto, foram analisados criticamente.

\section{Trabalhos selecionados}

Foram selecionados três trabalhos' ${ }^{1,4} 4$. Os trabalhos excluídos 2, 5-9 tiveram como motivo: desfecho analisado intermediário ${ }^{5}$, diferenças prognósticas no baseline, como classe do NYHA diferentes para o grupo de intervenção e controle ${ }^{6-9}$ e pacientes estudados que não correspondiam aos pacientes de interesse ${ }^{2}$.

\section{Sobrevida}

Há controvérsia quanto à diferença de sobrevida entre a cirurgia de revascularização do miocárdio associada ou não à correção da valva mitral, na dependência do grau de insuficiência, com resultados de redução não-significativa na mortalidade em 1, 5 ou 10 anos de seguimento, em pacientes grau moderado a importante, e resultados de redução significativa de mortalidade, em 2 e 3 anos, de 15,8\%(IC95\% 0,3\% a $31,3 \%$ e 19,6\%(IC95\% I,9\% a 37,3\%), respectivamente, em pacientes grau médio a moderado. Reintervenção a partir de I,5 anos: redução não-significativa na necessidade de reintervenção nos pacientes graus médio a moderado, submetidos à cirurgia combinada ${ }^{4}$. Conclusão: Embora a associação da correção de valva mitral à revascularização miocárdica não altere a mortalidade do paciente grau moderado a importante, em I, 5 e 10 anos, houve evidência de que há um aumento na sobrevida em 2 e 3 anos de seguimento, em paciente grau médio a moderado. Entretanto, havendo indicação absoluta de troca de valva mitral, a associação da revascularização à substituição valvar deve ser realizada, uma vez que não há aumento da mortalidade se ambas forem feitas concomitantemente.

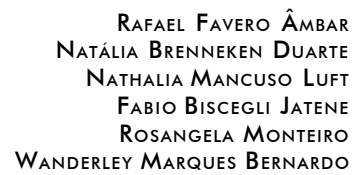

Referências

I. Mihaljevic T, Lam BK, Rajeswaran J, Takagaki M, Lauer MS, Gillinov AM, et al. Impact of mitral valve annuloplasty combined with revascularization in patients with functional ischemic mitral regurgitation. J Am Coll Cardiol 2007; 49: $2191-201$.

2.Diodato MD, Moon MR, Pasque MK, Barner HB, Moazami N, Lawton JS, et al. Repair of ischemic mitral regurgitation does not increase mortality or improve long-term survival in patients undergoing coronary artery revascularization: a propensity analysis. Ann Thorac Surg 2004; 78: 794-9.

3.Kang DH, Kim MJ, Kang SJ, Song JM, Song H, Hong MK, et al. Mitral valve repair versus revascularization alone in the treatment of ischemic mitral regurgitation. Circulation 2006; I | 4( I Suppl): 1499-503.

4.Prifti E, Bonacchi M, Frati G, Giunti IG, Leacche M, Proietti P, et al. Should mild-to-moderate and moderate ischemic mitral regurgitation be corrected in patients with impaired left ventricular function undergoing simultaneous coronary revascularization? J Card Surg 200 I; 16: 473-83.

5.Di Donato M, Frigiola A, Menicanti L, Boghdabi A, Badia T, Neagu A, et al. Moderate ischemic mitral regurgitation and coronary artery bypass surgery: effect of mitral repair on clinical outcome. J Heart Valve Dis 2003; 12: 272-9. 6. Herlitz J, Brandrup-Wognsen G, Caidahl K, Haglid M, Karlsson BW, Karlsson $\mathrm{T}$, et al. Mortality and morbidity among patients who undergo combined valve and coronary artery bypass surgery: early and late results. Eur J Cardiothorac Surg 1997; 12: 836-46.

7. Harris KM, Sundt TM 3rd, Aeppli D, Sharma R, Barzilai B. Can late survival of patients with moderate ischemic mitral regurgitation be impacted by intervention on the valve? Ann Thorac Surg 2002; 74: I468-75.

8.Trichon BH, Glower DD, Shaw LK, Cabell CH, Anstrom KJ, Felker GM, et al. Survival after coronary revascularization, with and without mitral valve surgery, in patients with ischemic mitral regurgitation. Circulation 2003; 108 Suppl I: III03-10.

9.Isomura T, Suma H, Yamaguchi A, Kobashi T, Yuda A. Left ventricular restoration for ischemic cardiomyopathy-comparison of presence and absence of mitral valve procedure. Eur J Cardiothorac Surg 2003; 23: 6I 4-9. 